\title{
Article
}

\section{Loneliness, HPA Stress Reactivity and Social Threat Sensitivity: Analyzing Naturalistic Social Challenges}

Nowland, Rebecca, Robinson, Sarita Jane, Bradley, Belinda Fay, Summers, Vicki and Qualter, Pamela

Available at http://clok.uclan.ac.uk/23042/

Nowland, Rebecca ORCID: 0000-0003-4326-2425, Robinson, Sarita Jane ORCID: 0000-0002-4237-5412, Bradley, Belinda Fay ORCID: 0000-0002-34268924, Summers, Vicki and Qualter, Pamela (2018) Loneliness, HPA Stress Reactivity and Social Threat Sensitivity: Analyzing Naturalistic Social Challenges. Scandinavian Journal Of Psychology . ISSN 0036-5564

It is advisable to refer to the publisher's version if you intend to cite from the work. http://dx.doi.org/10.1111/sjop.12461

For more information about UCLan's research in this area go to http://www.uclan.ac.uk/researchgroups/ and search for <name of research Group>.

For information about Research generally at UCLan please go to http://www.uclan.ac.uk/research/

All outputs in CLoK are protected by Intellectual Property Rights law, including Copyright law. Copyright, IPR and Moral Rights for the works on this site are retained by the individual authors and/or other copyright owners. Terms and conditions for use of this material are defined in the policies page. 
RUNNING HEAD: Loneliness \& stress reactivity

Loneliness, HPA Stress Reactivity and Social Threat Sensitivity: Analyzing Naturalistic Social Challenges

Author Accepted Manuscript

Other: Rebecca Nowland, Sarita J. Robinson, Belinda F. Bradley, Vicki Summers, and Pamela Qualter Loneliness, HPA, Stress Reactivity, and Social Threat Sensitivity: Analyzing Naturalistic Social Challenges. Scandinavian Journal of Psychology.

This is the author accepted manuscript. It is advisable to refer to the publisher's version of the paper if you intend to cite from the work. 
RUNNING HEAD: Loneliness \& stress reactivity

\begin{abstract}
Loneliness has been linked to poor health through an increased activation of threat surveillance mechanisms, such as the hypothalamic-pituitary-adrenal axis (HPA). The socio-cognitive model (Cacioppo \& Hawley, 2009) proposes that lonely people have an increased social threat sensitivity which activates the HPA axis. The current study examined the impact of loneliness on HPA stress reactivity and social threat sensitivity in response to naturally occurring social challenges. Participants $(\mathrm{N}=45)$ were prospective undergraduates attending a 3-day university preparation programme over the summer, prior to commencing their university studies. Cortisol levels and perceived stress were measured before and after an ice breaker session on Day 1 and a lecture session on Day 3. Social threat sensitivity was also measured on the first and third day. When meeting unfamiliar peers in the ice breaker session, HPA stress reactivity was evident, but it was not markedly different in those who reported high levels of loneliness than those with low levels. The high loneliness group had higher levels of perceived stress and increased social threat sensitivity than the low loneliness group on both testing days. The findings show partial support for the socio-cognitive model of loneliness because increased threat sensitivity was demonstrated in the high loneliness group. The findings indicate that lonely people do not respond in a physiologically different way to specific social challenges, but they typically report higher social threat sensitivity and higher perceived stress than their non-lonely peers.
\end{abstract}

Key words: loneliness, friendships, social threat, threat sensitivity, hyperviligance to social threat, cortisol, stress, ice breaker 
RUNNING HEAD: Loneliness \& stress reactivity

Funding: This research did not receive any specific grant from funding agencies in the public, commercial or not-for-profit sectors. The work was supported by a university funded PhD studentship awarded to the first author. 
RUNNING HEAD: Loneliness \& stress reactivity

Loneliness is an aversive state that is experienced when a person perceives that the social connections they have do not meet their needs (Peplau \& Perlan, 1982). This is an important mood state that promotes reconnection with others, but when prolonged, loneliness is linked to poor physical and mental health (Shiotiz-Ezra \& Ayalon, 2010). One mechanism by which loneliness is proposed to have an impact on health is an increased perception of social threat, which not only perpetuates feelings of loneliness, but also increases the load on threat surveillance mechanisms, contributing to poor health (Cacioppo \& Hawkley, 2009). In particular, the hypothalamic-pituitary-adrenal axis (HPA) has been implicated as a threat surveillance mechanism involved in the relationship between perceived social threat relating to loneliness and poor health (Cacioppo, Cacioppo, Capitanio, \& Cole, 2015). This threat surveillance mechanism could lead to poor health in two different ways: 1) by a chronic activation of the HPA axis leading to dysregulation of HPA functioning demonstrated by atypical diurnal cortisol patterns (Miller \& O’Callaghan, 2002) and/or 2) by increased HPA stress reactivity to social stressors (Schlotz, Hammerfald, Ehlert, \& Gabb, 2011) as a result of an increased perception of social threat.

In relation to the first proposition, there is evidence that lonely people have a dysfunction of the normative cortisol pattern across a typical day: lonely people have a higher cortisol awakening response, increased mean levels of cortisol, and a flattening of the diurnal cortisol slope (Cacioppo, et al., 2000; Doane \& Adam, 2010; Steptoe, Owen, Kunz-Ebrecht, \& Brydon, 2004). Also, short term increases in loneliness are associated with steeper cortisol slopes (Drake, Sladek, \& Doane, 2015) indicating that the state of loneliness leads to alterations in levels of cortisol. 
RUNNING HEAD: Loneliness \& stress reactivity

The second proposition is based on the stress reactivity hypothesis (Scholtz et al., 2011) which argues that there are individual differences in the stress response. It follows that lonely people who perceive increased levels of potential threat in a social situation (Cacioppo \& Hawkley, 2009) will have higher stress reactivity in social situations than non-lonely people. A recent survey of empirical studies that have examined acute physiological responding in lonely people showed that loneliness is associated with an atypical physiological response (Brown, Gallagher, \& Ceaven, 2017). Some of these studies reviewed measured the cardiovascular reactivity and others have measured neuroendocrine reactivity (i.e HPA response). Only a few studies of those studies examined HPA stress reactivity directly. Using non-social stressor tasks, one study found that HPA stress responses were small and not related to loneliness (Steptoe et al., 2004) and in another lower HPA stress reactivity was found in lonely women but not men (Hackett, Hamer, Endrighi, Brydon, \& Steptoe, 2012). When a social stress task was used (i.e. a public speech task), no association between loneliness and HPA stress reactivity was found (Edwards, Bosch, Engeland, Cacioppo, \& Marucha, 2010).

The contribution of these studies to our understanding of how loneliness impacts on HPA stress reactivity are limited for three reasons: 1) social tasks have not always been used, 2) the studies have been conducted in laboratory conditions, and 3) social threat sensitivity has not been measured. Firstly, the use of social tasks is important because the HPA has a specific role in social stress (Dickerson \& Kemeny, 2010). It is difficult to be conclusive about that evidence because social stress tasks were always not used. Thus, it is important to establish whether the contradictory findings of existing stress reactivity studies are the result of not using a social task. Secondly, what is also missing from the extant literature is an examination of stress reactivity in response to a real life social stressor because all the studies to date have been carried out in a laboratory. It is important to examine lonely people's response in real life situations because these may differ from an artificial laboratory scenario and the absence of 
RUNNING HEAD: Loneliness \& stress reactivity

HPA stress reactivity in such studies may be the result of the situation being a controlled and unnatural one.

Thirdly, although the socio-cognitive model predicts that lonely people will have an increased perception of social threat, to date this has not been measured in a real life social situation nor has social threat sensitivity been measured alongside HPA axis stress reactivity. An increased perception of social threat in lonely people has only been demonstrated in laboratory tasks (e.g. Bangee, Harris, Bridges, Rotenberg, \& Qualter, 2014; Qualter et al. 2013; Vanhalst et al., 2013). To the authors' knowledge there are no measures to examine social threat sensitivity in situ; measures exist only for social evaluation anxiety more generally (i.e. typical anxiety, rather than specific to a particular social situation). In the current study in order to examine social threat sensitivity to a specific social challenge a social threat sensitivity measure was devised by the authors.

Subjective levels of stress have not always been measured in loneliness and stress reactivity studies, but when measured, lonely people have increased levels of perceived stress in the non-testing periods (Hackett et al., 2012), indicating that lonely people have a higher perception of stress generally rather than in a response to a socially stressful situation. This proposition is supported by evidence from diary studies that show that lonely people generally report higher levels of perceived stress and negative affect than non-lonely people in everyday life (Cacioppo et al., 2000; Van Roekel, Goossens, Verhagen, Wouters, Engels, \& Scholte, 2013). Lonely people do not experience an increased number of stressful events, but tend to rate these events as more stressful than non-lonely people (Cacioppo et al., 2000; Steptoe et al. 2004). In the current study we measure perceived stress alongside physiological stress reactivity because that evidence indicates that results obtained from subjective measures of stress would be different to those when physiological measures of stress are used. 
RUNNING HEAD: Loneliness \& stress reactivity

There is evidence to suggest that the social challenge of meeting new people may be a particularly stressful scenario for lonely people. First, loneliness has been associated with an atypical social information processing style, involving self-defeating behaviour, such that lonely people tend to make negative and hostile attributions to others, expect rejection by others, and evaluate themselves and others negatively (Spithoven, Bijttbier, \& Goosens, 2017). Second, a few empirical studies have examined behaviour of lonely people when meeting others in a laboratory setting and have found that they engage in more self-defeating behaviour. When people were paired with strangers to complete a "getting to know you exercise”, lonely people engaged in less self-disclosure and partner attention than non-lonely people, indicating they are more guarded when meeting new people (Jones, Hobbs, \& Hockenbery, 1982; Solano, Batten, \& Parish, 1982). Lonely people also tend to interpret their own and their social partners behaviour negatively in social encounters and expect others to rate them negatively (Duck, Pond, \& Leatham, 1994; Jones, Sansone, \& Heim, 1983). The current study uses a real life scenario where people are meeting others for the first time. Two sessions are used: one where participants are actively involved in ice breakers and another session where participants are in an introductory lecture session. It is expected that the ice breaker session will be more social challenging because students are actively involved in "getting to know you” activities.

The current study

The current study addresses the gaps in the extant literature on loneliness and stress by measuring HPA stress reactivity, perceived stress, and perception of social threat sensitivity in a two social challenges: meeting unfamiliar peers in an icebreaker (moderate social challenge) and attendance at a lecture (mild social challenge) during a 3-day orientation to university course. We predict that lonely people will report higher levels of social threat sensitivity and 
RUNNING HEAD: Loneliness \& stress reactivity

perceived stress and have an increased stress reactivity to the naturally occurring social challenges.

Method

Participants

Participants were recruited from a group of prospective undergraduate students $(\mathrm{N}=$ 397) attending one of three pre-university orientation courses taking place in a UK university in a North West region in 2011. These 3-day orientation courses were designed to introduce students to the university and their peers during the summer prior to starting university in the October (i.e. they had not commenced their university studies yet). This particular population was chosen for the study because they all would be meeting people for the first time and involved in "getting to know you" activities. Participants were recruited across a wide range of disciplines within the university. All prospective undergraduate students that were planned to attend the orientation course were invited to take part in the study via a letter that was sent to their homes before the course commenced. Sixty-seven of those invited expressed an interest to take part in the study (i.e. by returning a slip with contact details); of those, only 48 took part in the study on the data collection days. From those 48 participants, one was unable to participate because they did not pass the medical screening, and two others were not present at the data collection times. The remaining 45 participants were aged between 17-46 years (mean age $=20.24 ; \mathrm{SD}=5.33,66.70 \%$ female) and were prospective undergraduate students from the following disciplines: psychology, neuroscience and counselling (27\%), media, fashion and design (19\%), health/exercise (8\%), humanities (5\%), languages (5\%), and law (3\%)

\section{Self report measures}


RUNNING HEAD: Loneliness \& stress reactivity

Loneliness was measured using the 20 item R-UCLA loneliness scale (Russell, 1996). Participants were asked to rate how they usually feel in response to a list of statements such as "I feel in tune with the people around me” and "I lack companionship” on a Likert scale ranging from 1 (never) to 4 (often). After reverse scoring the relevant statements, a loneliness score for each participant was calculated by summing all the statements. Possible scores range from 20 to 80, with higher scores signifying greater loneliness. This scale showed good internal consistency in the current sample ( $\alpha=.92)$.

Depressive symptoms were assessed using the Centre for Epidemiologic Studies Depression Scale (CES-D; Radloff, 1977). The CES-D is a self-report measure used to check for the presence and persistence of depression symptoms. The questionnaire contains 20 items (16 negative statements and 4 positive statements which were reverse coded) describing a state of mind. For example, 'I was bothered by things that usually don't bother me' and 'I felt fearful'. Participants were asked to consider how many days over the last week they agreed with each item, using a 4-point Likert scale from $1=$ rarely (less than one day) through to $4=$ most of the time (5-7 days). A high score was indicative of a high presence of depressive symptoms, with a possible range of scores of between 0-60. A score of over 27 was taken as an indication of clinical levels of depression (Boyd, Wiessman, Thompson, \& Myers, 1982; Zich, Attkisson, \& Greenfield, 1990). High internal consistency was shown for this measure in the current study $(\alpha=.87)$.

Perceived Stress was measured using the stress subscale on the Stress and Arousal checklist (SACL; Mackay, Cox, Burrows, et al., 1978). The stress subscale uses 11 positive adjective mood-related words, such as 'Peaceful' and 'Relaxed' and 8 negative adjective moodrelated words, such as 'Worried' and 'Tense'. Participants were asked to select the word which best describes their current state from the options: 'Definitely Feel', Slightly Feel', 'Cannot 
RUNNING HEAD: Loneliness \& stress reactivity

Decide' and 'Definitely Do Not Feel'. A score of 0 was given when a person selected 'Definitely Feel' or 'Slightly Feel' for positive adjectives and a score of 1 was given when participants select 'Cannot Decide' or 'Definitely Do Not Feel'. For the negative adjectives scoring was reversed. The maximum score on the stress sub-scale was 19 with higher scores representing higher perceived stress. An acceptable level of internal consistency was shown for the stress sub-scale in this study (average $\alpha=.85$ ).

Social Threat Sensitivity was measured using a scale devised by the authors comprising the following items: "How anxious do you feel about taking part in the session?” "How anxious do you feel about meeting people in the session?" and "How anxious do you feel about how other people in the group may perceive your participation in the session?” with participants responding on a 7 point Likert scale from 1 (not anxious at all) to 7 (extremely anxious) and "how likely do you think other people in the group may perceive your performance positively" rated on the scale of 1 (not very likely) to 7 (very likely). And a final question that was reverse coded, "How much do you think that your participation in the session today will have a positive effect on your friendships in the group?” which was rated by participants on a scale from 1 (negative effect) to 7 (positive effect). This measure demonstrated high internal consistency; average $\alpha=.78$. Given that this measure used was created by the authors for the purposes of the study, to examine its validity, it was correlated with a measure of generalised social anxiety (Fear of Negative Evaluation; Watson \& Friend, 1969) taken on the first day and correlated well with the standardised measure of generalised social anxiety (Day $1-\mathrm{r}=.48, \mathrm{p}=.002$ and Day $3-\mathrm{r}=.53, \mathrm{p}<.001)$.

\section{HPA stress reactivity}


RUNNING HEAD: Loneliness \& stress reactivity

Participants were instructed to give unstimulated saliva samples by placing a salivette (Sarstedt Ltd, Leicester, UK) in their mouth until it was saturated. Participants were asked to refrain from smoking, eating, or drinking during the testing session (with the exception of drinking water). Samples were stored at $-20^{\circ} \mathrm{C}$ and were recovered by thawing at room temperature, then centrifuging (1500 rpm) for 15 minutes. Cortisol concentration (nmol/l) was determined by a high sensitivity salivary cortisol enzyme-linked immunosorbent assay kit in accordance with the manufacturer's instructions (Salimetrics, USA), using the Perkin Elmer JANUS automated liquid handling system. Intra-assay variation was acceptable with a variation coefficient of less than $10 \%$. Any cortisol samples that were 3 standard deviations from the mean were removed from the analyses, resulting in removal of 5 samples from the overall set of 252 .

\section{Procedure}

Participants were recruited prior to the start of the 3-day programme and completed a questionnaire pack comprising loneliness, depression, social anxiety and medical screening questions. All participants were screened for depression and existing medical conditions that may affect cortisol measurement. Three participants were removed because they had clinical levels of depression. The data for the remaining 42 participants were used in the analyses.

The researchers selected an activity at the beginning of the week where students were involved in ice breakers with their peers (moderate social challenge) and a lecture session on the final day (mild social challenge). Data collection took place on Day 1 and Day 3 of the 3day programme. Social threat sensitivity was completed once on Day 1 prior to the ice breaker session and once on Day 3 prior to the lecture session. Saliva samples and perceived stress measures were taken at three time points on both days (Time $1=$ immediately before the 
RUNNING HEAD: Loneliness \& stress reactivity

session, Time 2 = immediately after the session, and Time $3=20$ minutes after the session). Both sessions took place before lunch and lasted one hour. Due to restrictions on when activities were taking place, on Day 1 the first saliva sample (Time 1) was at approximately $10 \mathrm{am}$ and 12 noon on Day 3.

Ethics

All participants gave written consent and were tested in accordance with the national and local ethics guidelines. The study was approved by the University Ethics Review Board. All measures, manipulations, and exclusions in the studies are disclosed, as well as the method of determining the final sample size.

Data analysis Plan

For all analyses participants were grouped into high and low loneliness groups based on their loneliness scores using median split (Cacioppo et al., 2002; Steptoe et al., 2004). The low loneliness group scored below $39(\mathrm{~N}=21, \mathrm{~F}=15)$ and the high loneliness group scored above 40 ( $\mathrm{N}=21, \mathrm{~F}=15)$ and loneliness scores were significantly different between the groups $(\mathrm{t}(43)=8.58, \mathrm{p}<.001)$. The age range for the low loneliness group was 18-46 years (mean age $=20.10, \mathrm{SD}=6.28$ ) and 17-33 years for the high loneliness group (mean age $=21.06$, SD $=5.18$ ). One person in the low loneliness group was aged 46 years old (all other participants were in the age range 18-35 years). In order to ensure that there was no effect of this person on the results due to an increased age from the rest of the group (17-33 years old), analyses were conducted without this person and results remained the same. An independent t-test 
RUNNING HEAD: Loneliness \& stress reactivity

revealed that there were not significant differences in age between the loneliness groups (t(38) $=0.08, \mathrm{p}=.934$ ) or gender (i.e. 15 females in each group of 21 participants).

A series of 3 (Time) x 2 (Loneliness group) x 2 (Day) ANOVAs were conducted for each of the measures. As it would be expected that cortisol levels would differ across the days because the time of data collection was different, cortisol data was analysed separately for each day. For the social threat sensitivity a 2 (Loneliness group) x 2 (Day) ANOVA was conducted because this was measured only once each day. In order to address whether HPA stress reactivity was associated with perceived stress and/or threat sensitivity and/or loneliness and depression correlation analyses were also conducted.

Results

Mean scores and standard deviations with ANOVA results for each measure are displayed in Table 1.

Cortisol

There were no significant main effects of loneliness group on either day, indicating that there were no differences in HPA stress reactivity between the high and low loneliness groups. There was a significant main effect of time on cortisol on both days. Significant post hoc comparisons are demonstrated in Figure 1 (Day 1) and Figure 2 (Day 3).

On Day 1, cortisol levels were significantly higher at Time 2 than Time $1(\mathrm{t}(38)=2.40$, $\mathrm{p}=.011$, one-tailed), indicating that the ice-breaker was sufficiently stressful to evoke a HPA stress response (Kudielka, Hellhammer \& Wust, 2004). There was a significantly lower level 
RUNNING HEAD: Loneliness \& stress reactivity

of cortisol at Time 3 than Time $2(\mathrm{t}(37)=1.94, \mathrm{p}=.030$, one-tailed $)$ indicating that stress recovery had occurred (Kudielka et al., 2004).

On Day 3, cortisol levels at Time 1 were significantly higher than Time $3(\mathrm{t}(32)=7.14$, $\mathrm{p}<.001)$, but not Time $2(\mathrm{t}(27)=1.38, \mathrm{p}=.181)$. Further, cortisol levels were significantly higher at Time 2 compared to Time $3(\mathrm{t}(24)=3.08, \mathrm{p}=.005)$. These results indicate that cortisol levels were higher prior to the lecture session, but reduced over time, displaying typical circadian decreases (King \& Hegadoren, 2002).

Cortisol levels on Day 3 were higher than on Day 1 despite the later data collection time which may reflect the impact of students' activities as part of the university orientation programme, for example, staying up late, lacking sleep.

\section{Social Threat Sensitivity}

There was a significant main effect of day and loneliness group for social threat sensitivity. Both groups had a reduction in their social threat sensitivity from Day 1 to Day 3, but the high loneliness group reported higher levels of social threat sensitivity on both days. There was no significant main effect of time on levels of social threat sensitivity and there were no interaction effects.

\section{Perceived Stress}

There was a significant main effect of day on perceived stress which shows that perceived stress was higher on Day 1 than Day 3. There was a significant main effect of loneliness group which shows that the high loneliness group reported more stress than the low loneliness group on both days. There was no significant main effect of time on levels of perceived stress and there were no interaction effects. 
RUNNING HEAD: Loneliness \& stress reactivity

Associations between HPA stress reactivity and self-report measures

In order to examine relationships between HPA stress reactivity, social threat sensitivity and perceived stress a measure of HPA stress reactivity was calculated by subtracting the cortisol levels for Time 2 from the cortisol levels from Time 1 on Day 1. Perceived stress was averaged across the measures. There was no significant association between the HPA stress reactivity and social threat sensitivity $(r=-.14)$ or averaged perceived stress $(r=-.06)$. This analysis was conducted for Day 3 as HPA stress reactivity was not evident on this day.

Associations between loneliness, depressive symptoms, and study variables on Day 1

To further examine relationships bivariate correlations between the study variables on Day 1 (where HPA stress reactivity) were conducted (see Table 2). Mean cortisol and perceived stress were calculated by averaging measurements at time points. Loneliness was moderately correlated with mean perceived stress and social threat sensitivity, but not mean cortisol on Day 1. Depressive symptoms and loneliness were moderately correlated and depression was also correlated with mean perceived stress, but not social threat sensitivity on Day 1.

\section{Discussion}

The current study examined whether people with high loneliness have increased HPA stress reactivity, social threat sensitivity and perceived stress in response to naturally occurring social challenges. Results show that the social challenge of meeting unfamiliar peers in an ice breaker session was sufficient to elicit HPA stress reactivity, but not the lecture session. There 
RUNNING HEAD: Loneliness \& stress reactivity

were no differences in HPA stress reactivity between the high and low loneliness groups, but the high loneliness group reported higher perceived stress and social threat sensitivity on both days. Increased social threat sensitivity and perceived stress was not associated to HPA stress reactivity on the ice breaker day.

The results in the current study are similar to previous laboratory studies which have found no difference in HPA stress reactivity between lonely and non-lonely people (Edwards et al., 2013; Hackett et al., 2012; Steptoe et al., 2004). The findings of previous HPA stress reactivity studies were limited as they did not always use social stress tasks and were all performed in a laboratory. The findings in the current study indicate that when the limitations of the previous studies are addressed, increased HPA stress reactivity to specific social challenges is not evident in lonely people.

As there were differences in perceived stress and social threat sensitivity in lonely people the results support the proposition that lonely people have a hyperviligance for social threat (Cacioppo \& Hawkley, 2009). Previous studies have measured perception of social threat only in laboratory scenarios but the current study is the first to demonstrate this in lonely people in a naturally occurring real life social situation. Perceived stress was also found to be higher in the high loneliness group in all testing periods, which is similar to previous studies that have shown that lonely people typically report increased levels of perceived stress in everyday life (Hackett et al., 2012; Hawkley et al. 2009). The socio-cognitive model (Cacioppo \& Hawkley, 2009) also proposes that an increased perception of social threat is associated with activation of HPA axis. In the current study neither perceived stress nor social threat sensitivity were related to HPA stress reactivity.

Strengths and limitations 
RUNNING HEAD: Loneliness \& stress reactivity

This is the first study to demonstrate HPA stress reactivity when meeting unfamiliar peers in adulthood. The findings show that, similar to children (Brotman et al., 2007; Granger, Stansbury, \& Henker, 1992), the social challenge of meeting unfamiliar peers also elicits HPA stress reactivity in adulthood. It is also the first study to examine the relationship between loneliness and HPA stress reactivity using a naturalistic social stressor. Although it is a strength of the current study that a naturalistic social challenge was used, the study did not use a standardised protocol to elicit social stress therefore the absence of a difference in the loneliness groups may be due to the task being sufficiently stressful to mount a stress response but not stressful enough to elicit HPA stress reactivity differences in lonely people.

In an attempt to use in a naturalistic setting the current study has some weaknesses in design that should be addressed in future studies. One such weakness is that there was not an examination of baseline days (e.g. typical days), so it is difficult to establish from the findings whether there were differences in cortisol functioning in these two atypical days (e.g. when attending a 3-day programme) in comparison to normal days. In some studies (Doane \& Adam, 2010; Steptoe et al., 2004) an increased cortisol awakening response (CAR) has been found in lonely people. It may be that the social challenging days evoked changes in the CAR for lonely people but given limitations in data collection this was not measured in this study. A further weakness of collecting data in a naturalistic setting is the sample size achieved in the current study. It is particularly challenging to recruit participants when they are already in a socially stressful situation because lower levels of consent to take part are expected (i.e. people may not want to increase their levels of stress or perception of being evaluated by others, which they may already be anxious about, by taking part in a research study). Future studies examining naturally occurring stressors should aim for larger sample sizes strategies by adopting strategies to overcome these challenge of collecting data in a naturalistic setting. 
RUNNING HEAD: Loneliness \& stress reactivity

The study recruited an undergraduate sample from a wide variety of disciplines, rather than from one study area. However, there are factors which make undergraduates a rather homogenous group. Undergraduate students may differ from other populations in a number of ways (e.g. young and highly educated) and results may not be replicated in other populations,

such as an aging population. In addition, the data were only collected in one UK based university so it is not clear if the findings can be generalised to undergraduates attending university in other parts of the world or in other UK universities.

In the current study, there were similar numbers of females in the high and low loneliness groups, so it is unlikely that gender impacted on the results in the current study because it involved an examination of differences between the lonely groups and gender composition was the same in both groups. However, it could be argued that there was an overrepresentation of females in the groups and similar results may not be obtained for males. Future studies could ensure that there is a sufficient sample size to allow examination of gender differences.

\section{Conclusion}

The current study found no differences in HPA stress reactivity between high and low loneliness groups in response to naturally occurring social challenges. But the high loneliness group reported higher perceived stress and increased social threat sensitivity in all testing periods. Such findings are important because they suggest that lonely people are not more reactive to socially challenging situations, but typically have higher levels of perceived stress and are generally on a heightened state of alert for social threat. 
RUNNING HEAD: Loneliness \& stress reactivity

\section{References}

Bangee, M., Harris, R. A., Bridges, N. J., Rotenberg, K., \& Qualter, P. (2014). Loneliness and attention to social threat in young adults: Findings from an eye tracker study. Personality and Individual Differences, 63, 16-23. doi: 10.1016/j.paid.2014.01.039

Boyd, J. H., Wiessman, M. M., Thompson, D., \& Myers, J. (1982). Screening for depression in a community sample: Understanding discrepancies between depression symptom and diagnostic scales. JAMA Psychiatry, 39, 1195-1200. doi:10.1001/archpsyc.1982.04290100059010.

Brotman, L.M., Gouley, K. K., Huang, K., Kamboukos, D., Fratto, C., Pine, D. S. (2007) Effects of a psychosocial family-based preventive intervention on cortisol response to a social challenge in preschoolers at high risk for antisocial behavior, Archives of General Psychiatry, 64, 1172-1179. doi:10.1001/archpsyc.64.10.1172.

Brown, E. G., Gallagher, S., \& Creaven, A. M. (2017). Loneliness and acute stress reactivity: A systematic review of psychophysiological studies. Psychophysiology. Advance online publication. doi:10.1111/psyp.13031

Cacioppo, J. T., Bernston, G. G., Malarkey, W. B., Kiecolt-Glaser, J. K., Sheridan, J. F., Poehlmann, K. M., Ernst, J. M., Hawkley, L. C., \& Glaser, R. (1998) Autonomic, neuroendocrine, and immune responses to psychological stress: The Reactivity Hypothesis. Annals of the New York Academy of Sciences, 840, 664-673. doi: 10.1111/j.1749-6632.1998.tb09605.x

Caccioppo, J. T., Cacioppo, S., Capitanio, J. P., \& Cole, S. W. (2015). The neuroendocrinology of social isolation. Annual Review of Psychology, 66, 733-67. doi: 10.1146/annurev-psych-010814-015240 
RUNNING HEAD: Loneliness \& stress reactivity

Cacioppo, J.T., Ernst, J. M., Burleston, M. H., McClintock, M. K., Malarkey, W. B., Hawkley, L. C., Kowalewski, R. B., Paulsen, A., Hobson, A., Hugdahl, K., Spiegel, D., \& Bernston, G. G. (2000). Lonely traits and concomitant physiological processes: The MacArthur social neuroscience studies. International Journal of Psychophysiology, 35, 143-154.

Cacioppo, J. T., \& Hawkley, L. C. (2009). Perceived social isolation and cognition, Trends in Cognitive Sciences, 13, 447-454. doi: 10.1016/j.tics.2009.06.005

Chida, Y., \& Steptoe, A. (2010). Greater cardiovascular responses to laboratory mental stress are associated with poor subsequent cardiovascular risk status: A meta-analysis of prospective evidence. Hypertension, 55, 1026-1032. doi:

\subsection{1/HYPERTENSIONAHA.109.146621}

Dickerson, S. S. \& Kemeny. M. E. (2004). Acute stressors and cortisol responses: A theoretical integration and synthesis of laboratory research. Psychological Bulletin, 130, 355-391. doi: 10.1037/0033-2909.130.3.355

Drake, E. C., Sladek, M. R., \& Doane, L. D. (2015). Daily cortisol activity, loneliness, and coping efficacy in late adolescence: A longitudinal study of the transition to college. International Journal of Behavioral Development, 40, 4334-345. doi:10.1177/0165025415581914

Doane, L. D. \& Adam, E. K. (2010). Loneliness and cortisol: Momentary, day-to-day, and trait associations. Psychoneuroendocriniology, 35, 430-441. doi: 10.1016/j.psyneuen.2009.08.005

Duck, S., Pond, K., \& Leatham, G. (1994). Loneliness and the evaluation of relational events. Journal of Social and Personal Relationships, 11, 253-276. doi: $10.1177 / 0265407594112006$ 
RUNNING HEAD: Loneliness \& stress reactivity

Edwards, Elevated Macrophage Migration Inhibitory Factor (MIF) is associated with depressive symptoms, blunted cortisol reactivity to acute stress, and lowered morning cortisol. Brain, Behavior \& Immunity, 24, 1202-1208. doi: 10.1016/j.bbi.2010.03.011

Granger, D. A., Stansbury, K., \& Henker, B. (1992). Preschoolers' behavioral and neuroendocrine responses to social challenge. Merrill-Palmer Quarterly, 40, 190-211.

Hackett, R. A., Hamer, M., Endrighi, R., Brydon, L., \& Steptoe, A. (2012). Loneliness and stress-related inflammatory and neuroendocrine responses in older men and women. Psychoneuroendocriniology, 37, 1801-1809. doi:10.1016/j.psyneuen.2012.03.016

Hawkley, L. C., \& Cacioppo, J. T. (2010). Loneliness matters: a theoretical and empirical review of consequences and mechanisms. Annals of Behavioral Medicine, 40, 218227. doi: 10.1007/s12160-010-9210-8

Jones, W. H., Hobbs, S. A., \& Hockenbury, D. (1982). Loneliness and social skills deficits. Personality Processes and Individual Differences, 42, 682-689. Doi: 10.1037/00223514.42.4.682

Jones, W.H., Sansone, C., \& Helm, B. (1983). Loneliness and interpersonal judgements. Personality and Social Psychology Bulletin, 9, 437-441. doi:10.1177/0146167283093014

Kudielka, B. M., Hellhammer, D. H., \& Wust, S. (2009). Why do we respond so differently? Reviewing determinants of human salivary cortisol responses to challenge. Psychoneuroendocrinology, 34, 2-18. 10.1016/j.psyneuen.2008.10.004

Mackay, C., Cox, T., Burrows, G., \& Lazzerini, T. (1978). An inventory for the measurement of self-reported stress and arousal. British Journal of Social and Clinical Psychology, 17, 283- 284. doi: 10.1111/j.2044-8260.1978.tb00280.x

Miller, D. B., \& O’Callaghan, J. P. (2002). Neuroendocrine aspects of the response to stress. Metabolism, 51, 5-10. doi: 10.1053/meta.002.33184 
RUNNING HEAD: Loneliness \& stress reactivity

Peplau, L. A. \& Perlman, D. (1982). Perspectives on loneliness. In L. A. Peplau \& D. Perlman, D. (Eds.). Loneliness: A Sourcebook of Current Theory, research and Therapy. (pp. 1-18). New York: NY:Wiley.

Radloff, L.S. (1977). The CES-D scale: A self report depression scale for research in the general population. Applied Psychological Measurement, 1, 385-401. doi: $10.1177 / 014662167700100306$

Russell, D. W. (1996). UCLA Loneliness Scale (Version 3): Reliability, validity, and factor structure. Journal of Personality Assessment, 66, 20-40. Doi: 10.1207/s15327752jpa6601_2

Schlotz, W., Hammerfald, K., Ehlert, U., Gabb, J. (2011). Individual differences in the cortisol response to stress in young healthy men: Testing the roles of perceived stress reactivity and threat appraisal using multiphase latent growth curve modelling. Biological Psychology, 87, 257-264 doi: 10.1016/j.biopsycho.2011.03.005

Shiovitz-Ezra, S \& Ayalon, L. (2010). Situational versus chronic loneliness as a risk factor for all-cause mortality. International Psychogeriatrics, 22, 455-462. doi: 10.1017/S1041610209991426

Solano, C. H., Batten, P. G., \& Parish, E. A. (1982). Loneliness and patterns of selfdisclosure. Journal of Personality and Social Psychology, 43, 524-531. Doi: doi.org/10.1037/0022-3514.43.3.524

Spithoven, A. W. M., Bijttebier, P., \& Goossens, L. (2017). It's all in their mind: A review of information processing bias in lonely individuals. Clinical Psychology Review, 58, 97-114. doi:10.1016/j.cpr.2017.10.003

Steptoe, A., Owen, N., Kunz-Ebrecht, S. R. \& Brydon, L. (2004). Loneliness and neuroendocrine, cardiovascular, and inflammatory stress response in middle-aged 
RUNNING HEAD: Loneliness \& stress reactivity

men and women. Psychoneuroendocrinology, 29, 593-611. doi: 10.1016/S03064530(03)00086-6

Qualter, P., Rotenberg, K. J., Barrett, L., Henzi, S. P., Barlow, A., Stylianou, M., \& Harris, R. A. (2013). Investigating the hyperviligance of social threat of loneliness in children. Journal of Personality and Social Psychology, 41, 325-338. Doi: 10.1007/s10802012-9676-X

Vanhalst, J., Soenens, B., Luyckx, K., Van Petegem, S., Weeks, M. S.; \& Asher, S. R. (2015). Why do the lonely stay lonely? Chronically lonely adolescents’ attributions and emotions in situations of social inclusion and exclusion. Journal of Personality and Social Psychology, 109, 932-948. doi: 10.1037/pspp0000051

Van Roekel, E., Goossens, L., Verhagen, M., Wouters, S., Engels, R. M. C. E., \& Scholte, R. H. J. (2013). Loneliness, affect, and adolescents’ appraisals of company: An experience sampling method study. Journal of Research on Adolescence, 24, 350363. doi: 10.1111/jora.12061

Watson, D, \& Friend, R. (1969). Measurement of social-evaluation anxiety. Journal of Consulting and Clinical Psychology, 33, 448-457. doi: 10.1037/h0027806

Zich, J. M., Attkisson, C. C. \& Greenfield, T. K. (1990). Screening for depression in primary care clinics: The CES-D and the BDI. The International Journal of Psychiatry in Medicine, 20, 259 - 277. doi: 10.2190/LYKR-7VHP-YJEM-MKM2 
RUNNING HEAD: Loneliness \& stress reactivity

Table 1. Means (and standard deviations) and ANOVA results for cortisol levels and perceived stress by loneliness group on Day 1 and Day 3

\begin{tabular}{|c|c|c|c|c|c|c|c|c|c|c|c|c|c|c|}
\hline & & \multicolumn{6}{|c|}{ Day 1} & \multicolumn{6}{|c|}{ Day 3} & \multirow[t]{3}{*}{ \#Main effects } \\
\hline & & \multicolumn{2}{|c|}{ High Lonely } & \multirow[b]{2}{*}{ T3 } & \multicolumn{3}{|c|}{ Low Lonely } & \multicolumn{2}{|c|}{ High Lonely } & \multirow[b]{2}{*}{ T3 } & \multicolumn{3}{|c|}{ Low Lonely } & \\
\hline & & $\mathrm{T} 1$ & $\mathrm{~T} 2$ & & $\mathrm{~T} 1$ & $\mathrm{~T} 2$ & Т3 & $\mathrm{T} 1$ & $\mathrm{~T} 2$ & & $\mathrm{~T} 1$ & $\mathrm{~T} 2$ & Т3 & \\
\hline Cortisol & $M$ & 4.46 & 6.51 & 5.44 & 5.07 & 7.78 & 5.63 & 10.67 & 7.91 & 6.17 & 12.56 & 9.23 & 5.65 & $D A Y 1$ \\
\hline$(\mathrm{n} / \mathrm{mol})$ & $S D$ & 2.46 & 5.77 & 3.85 & 2.30 & 7.58 & 3.67 & 6.06 & 8.90 & 3.72 & 5.30 & 5.89 & 3.09 & $\begin{array}{l}\text { LG - F }(1.55,54.57)=1.22, \mathrm{p}=.294, \eta \mathrm{p}^{2}=.03 \\
\text { TIME- } \mathrm{F}(1.55,54.57)=4.38, \mathrm{p}=.025, \eta \mathrm{p}^{2}=.11^{*} \\
\text { DAY3 } \\
\text { LG - F }(1,23)=0.10, \mathrm{p}=.750, \eta \mathrm{p}^{2}=.04 \\
\text { TIME- } \mathrm{F}(1.52,34.91)=8.10, \mathrm{p}=.001, \eta \mathrm{p}^{2}=.26^{* *}\end{array}$ \\
\hline Perceived & $M$ & 6.20 & 5.31 & 4.94 & 3.10 & 3.18 & 2.39 & 2.71 & 3.08 & 3.29 & 0.70 & 1.65 & 1.32 & LG - F(1,14) = 38.03, $p=.031, \eta p^{2}=.29$ \\
\hline stress & $S D$ & 3.49 & 4.32 & 2.88 & 4.05 & 3.94 & 4.03 & 1.83 & 4.15 & 3.22 & 0.73 & 1.69 & 1.25 & $\begin{array}{l}\text { DAY - F }(1,14)=11.67, p=.004, \eta p^{2}=.14^{* *} \\
\text { TIME }-F(2,28)=0.12, p=.891, \eta p^{2}<.01\end{array}$ \\
\hline Social & $M$ & 18.85 & & & 15.77 & & & 17.75 & & & 13.62 & & & $\begin{array}{l}\text { LG - } \mathrm{F}(1,36)=10.98, \mathrm{p}=.002, \eta \mathrm{p}^{2}=.23^{* *} \\
\text { DAY- } \mathrm{F}(1,36)=3649, \mathrm{p}<.001, \eta \mathrm{p}^{2}=.50^{* *}\end{array}$ \\
\hline sensitivity & $S D$ & 4.49 & & & 3.98 & & & 4.96 & & & 3.09 & & & \\
\hline
\end{tabular}

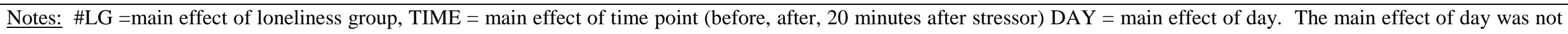
compared for cortisol because times of testing on each testing day was different. **significant at $\mathrm{p}<.01$, *significant at $\mathrm{p}<.05$ 
RUNNING HEAD: Loneliness \& stress reactivity

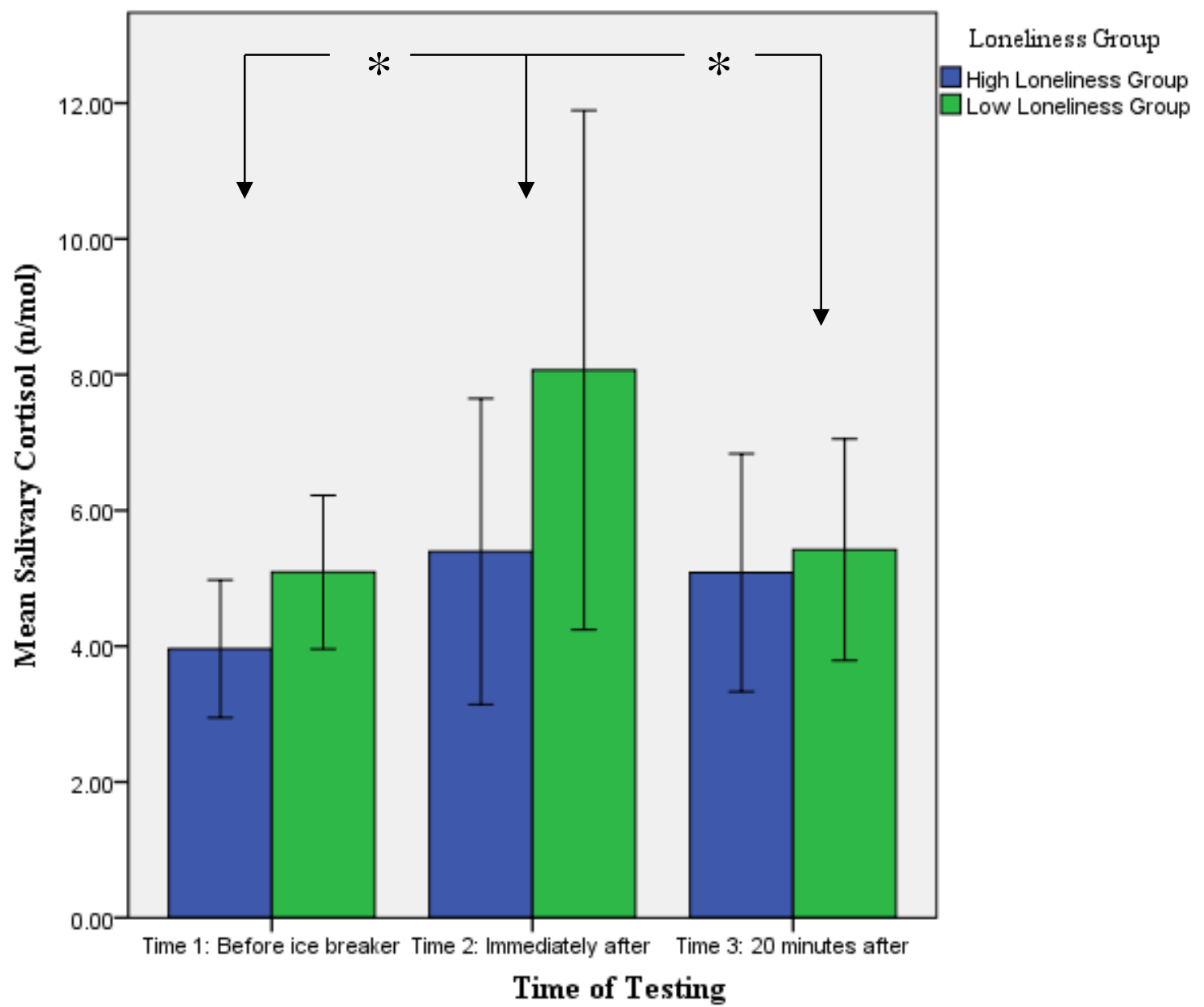

Note: *significant at $\mathrm{p}<.05$

Figure 1 . Day 1 cortisol levels before, immediately after and 20 minutes after the ice breaker session (with 95\% CI error bars) 
RUNNING HEAD: Loneliness \& stress reactivity

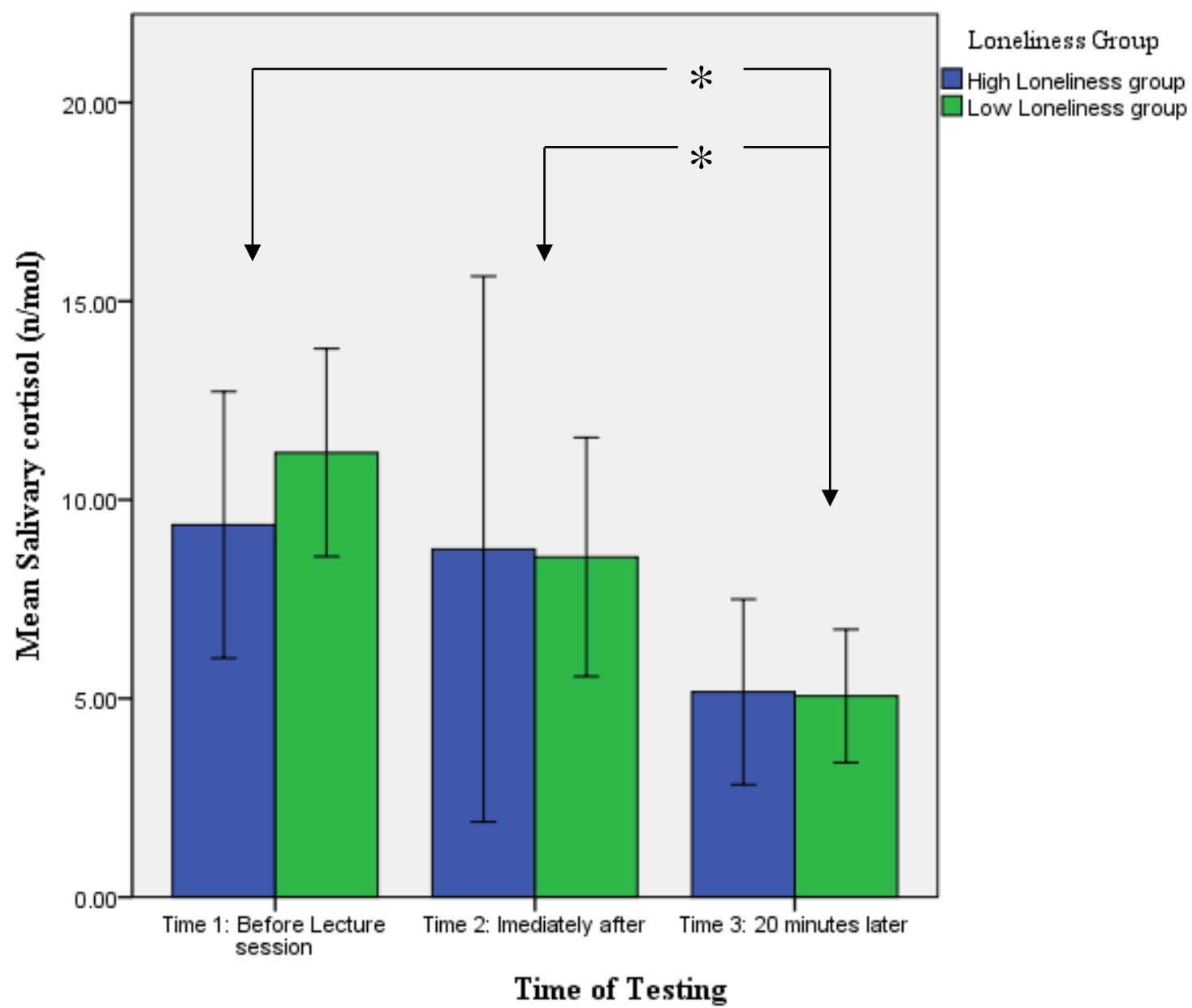

Note: *significant at $\mathrm{p}<.01$

Figure 2. Day 3 cortisol levels before, immediately after and 20 minutes after the lecture session 
RUNNING HEAD: Loneliness \& stress reactivity

Table 2. Bivariate correlations between study variables

\begin{tabular}{|c|c|c|c|c|c|c|}
\hline & Mean & SD & 2 & 3 & 4 & 5 \\
\hline Loneliness & 41.50 & 9.98 & $.34 *$ & $.44^{*}$ & .04 & $.44 * *$ \\
\hline Depression & 16.98 & 6.04 & & $.49 * *$ & -.15 & .19 \\
\hline Day 1 Mean Cortisol & 10.69 & 9.39 & & & -.17 & .33 \\
\hline Day 1 Mean Perceived stress & 5.48 & 3.46 & & & & -.10 \\
\hline Day 1 Social threat sensitivity & 12.92 & 4.15 & & & & \\
\hline
\end{tabular}

Note: Day 1 measures of cortisol and perceived stress have been averaged across each time point the measure was taken on that day. *significant at the $\mathrm{p}<.05$ level, ** significant at the $\mathrm{p}<.01$ level 